\title{
ORIGINAL ARTICLE \\ Text input speed in persons with cervical spinal cord injury
}

\author{
S Pouplin ${ }^{1,2,3,4}$, N Roche ${ }^{3,4,5}$, I Vaugier ${ }^{4}$, S Cabanilles ${ }^{1,2}$, C Hugeron ${ }^{2}$ and D Bensmail ${ }^{1,2,3,4}$
}

Study design: This is a prospective clinical study.

Objectives: The objectives of this study were to determine text input speed (TIS) in persons with cervical spinal cord injury (SCI) and to study the influence of personal characteristics and type of computer access device on TIS.

Setting: This study was conducted in the Rehabilitation Department, Garches, France.

Methods: People with cervical SCl were included if their level of injury was between $\mathrm{C} 4$ and $\mathrm{C} 8 \mathrm{Asia} \mathrm{A}$ or $\mathrm{B}$, and if they were computer users. In addition, able-bodied people were recruited from the hospital staff. Each participant underwent a single evaluation using their usual computer access devices. TIS was evaluated during a 10-min copying task. The relationship between the characteristics of participants with cervical SCI, type of computer access device and TIS were analyzed using a Scheirer-Ray-Hare test (nonparametric test similar to a two-way analysis of variance).

Results: Thirty-five participants with cervical SCI and 21 able-bodied people were included. Median TIS of participants with cervical $\mathrm{SCl}$ was $9(6 ; 14)$ words per minute (w.p.m.) and of able-bodied participants was 19 (14; 24)w.p.m. $(P=0.001)$. Median TIS of participants with lesions at or above C5 was $12(4 ; 13)$ w.p.m. and of those with lesions below C5 was $10(9 ; 18)$ w.p.m. $(P=0.38)$. The Scheirer-Ray-Hare test showed that only the type of computer access device significantly influenced TIS. Surprisingly, none of the person's characteristics, including the level of cervical lesion, affected TIS.

Conclusion: This is the first study to analyze TIS in a group of participants with cervical SCI. The results showed that only the type of computer access device influenced TIS.

Spinal Cord (2016) 54, 158-162; doi:10.1038/sc.2015.147; published online 15 September 2015

\section{INTRODUCTION}

The use of technological devices such as computers is often essential for the social and professional integration of persons with cervical spinal cord injury (SCI); ${ }^{1}$ however, they can be difficult to access. Depending on the level of the lesion, different devices exist to facilitate computer access. ${ }^{2}$ Persons with cervical SCI Asia A and B can be classified into two functional groups. ${ }^{3}$ The first group includes those whose injury level is at or above C5. To access a computer, this group requires assistive devices that are operated by head movements. The mouse cursor can be controlled by head tracking devices involving video or infrared cameras, ${ }^{4}$ and for text input, mouthsticks ${ }^{5,6}$ can be used with a standard keyboard or pointing devices with an on-screen keyboard. ${ }^{7,8}$

The second functional group includes those whose injury level is at or below C6 Asia A and B. This group may be able to use a standard keyboard with hand splints, or to press keys with the metacarpophalangeal joint of the little finger using forearm supination. Special devices such as trackballs and touchpads can be fitted to standard hardware to control the mouse cursor. Speech recognition devices are also an option for both groups. ${ }^{5}$

However, despite the development of these different devices, text input speed (TIS) remains lower for people with sensory motor impairments than for able-bodied people. ${ }^{9}$ In the literature, it is very difficult to find specific data regarding TIS for persons with cervical SCI, particularly for those with high levels of SCI (C5 or above, Asia A or B). Many studies have been carried out to evaluate TIS; ;,7,8 however, all of them have important limitations: first, the pathologies ${ }^{10}$ (both sensory and motor impairments such as lockedin syndrome, neuromuscular disorders and cervical SCI) and the severity of sensory-motor impairment ${ }^{11}$ (pooled results of assessments with persons with different motor function capacities) evaluated are quite heterogeneous. In addition, (i) the samples are usually very small; (ii) the computer access devices used very different; and (iii) some important methodological aspects are not specified. A summary of the studies evaluating TIS is provided in Table 1, which is classified according to the pathologies and devices studied.

The studies presented in Table 1 highlight the lack of data regarding the influence of participant's characteristics on TIS. The effect of lesion level, age, gender, education level, word processing use and duration of computer use have not been analyzed, although these parameters could influence TIS. ${ }^{12}$ A few studies in able-bodied people have assessed the effect of gender on TIS and showed an effect on Internet searching speed ${ }^{13}$ and on a visuospatial task ${ }^{14}$ in men. Until now, no study has evaluated the effects of such parameters in persons with SCI.

${ }^{1}$ New Technologies Plate-Form, AP-HP, Raymond Poincaré Teaching Hospital, Garches, France; ${ }^{2}$ Physical Medicine and Rehabilitation Department, AP-HP, Raymond Poincaré Teaching Hospital, Garches, France; ${ }^{3}$ Inserm Unit 1179, Team 3: Technologies and Innovative Therapies Applied to Neuromuscular Diseases, University of Versailles St-Quentinen-Yvelines, Versailles, France; ${ }^{4}$ Clinical Innovations Center 1429, AP-HP, Raymond Poincaré Teaching Hospital, Garches, France and ${ }^{5}$ Physiology-Functional Testing Ward, AP-HP, Raymond Poincaré Teaching Hospital, Garches, France

Correspondence: S Pouplin, Plate-Forme Nouvelles Technologies, Service de Médecine Physique et Réadaptation, Pavillon Widal Hôpital R Poincaré, 104 Boulevard R Poincaré, Garches 92380, France.

E-mail: samuel.pouplin@rpc.aphp.fr

Received 21 November 2014; revised 6 July 2015; accepted 9 July 2015; published online 15 September 2015 
Table $1 \mathrm{TIS}$ in the literature

\begin{tabular}{|c|c|c|c|c|}
\hline Participants & $\begin{array}{l}\text { Number of } \\
\text { participants }\end{array}$ & Devices & $\begin{array}{c}\text { TIS } \\
\text { (w.p.m.) }\end{array}$ & Studies \\
\hline \multirow{3}{*}{$\begin{array}{l}\text { Participants with } \\
\text { cervical SCl }\end{array}$} & $1-6$ & Standard keyboard & $5-35$ & $5-7,12$ \\
\hline & 1 & On-screen keyboard & $3-3$ & 7,15 \\
\hline & 1 & $\begin{array}{l}\text { Speech recognition } \\
\text { system }\end{array}$ & 20 & 5 \\
\hline \multirow{4}{*}{$\begin{array}{l}\text { Participants with } \\
\text { sensory motor impairment } \\
\text { (mixed pathologies) }\end{array}$} & $1-24$ & Standard keyboard & $1-38$ & $8,12,19$ \\
\hline & $1-10$ & On-screen keyboard & $0.5-24$ & $8,10,11$ \\
\hline & $1-8$ & $\begin{array}{l}\text { Scanning on-screen } \\
\text { keyboard }\end{array}$ & $0.3-5$ & 20,21 \\
\hline & $5-24$ & $\begin{array}{l}\text { Speech recognition } \\
\text { system }\end{array}$ & $5-113$ & 19,22 \\
\hline
\end{tabular}

Abbreviations: TIS, text input speed; SCl, spinal cord injury; w.p.m., words per minute.

Indeed, studies of people with cervical SCI have been limited to case studies or studies of small samples, ${ }^{5-7,12,15}$ focusing on TIS but not on the possible interaction between participant's characteristics and TIS.

It has also been shown that differences in TIS could be explained by the use of different computer access devices; 5,7 however, these studies were carried out on very small numbers of heterogeneous patients. Existing devices are considered to be adapted to the level of injury, functional capacities, sensory motor impairments and spasticity of people with SCI; however, the influence of individual devices on TIS has never been studied in a sample of people with SCI. Because these previous studies showed a very high level of variability of TIS and as typical TIS is not known, the aims of this study were first to study TIS in a sample of persons with Asia A or B tetraplegia and second to study (i) the influence of participant characteristics on TIS and (ii) the influence of the type of computer access device on TIS.

We hypothesized that participant characteristics and the type of computer access device would influence TIS in people with cervical SCI.

\section{MATERIALS AND METHODS}

\section{Participants}

This study was carried out between January 2011 and December 2012. During this period, participants with cervical SCI followed up in the Department of Physical Medicine and Rehabilitation of a Teaching Hospital were included if they were over 18 years old, had an SCI between C4 and C8 Asia A or B, were computer users and could read and write French. Participants were excluded if they had cognitive, linguistic or visual impairments. The study was approved by the local ethics committee (CPP Ile de France, Saint Germain en Laye, France), and all participants provided written informed consent before participation.

In addition, able-bodied people were recruited from hospital staff. The inclusion criteria were as follows: computer users who could read and write French. The able-bodied persons were matched to the participants with cervical SCI for age, gender and education level.

\section{Materials and study design}

To standardize the evaluation conditions, able-bodied participants all used a standard Hewlett-Packard Compaq 8510P computer (Hewlett-Packard, Palo Alto, CA, USA). The participants with cervical SCI used the same computer that was equipped with a Windows On-screen Keyboard and a speech recognition system (Dragon Naturally Speaking v.12, Nuance Society, Burlington, MA, USA), along with their usual computer access devices. If they usually used a speech recognition system, a standard keyboard or on-screen keyboard and pointing devices, the evaluations were carried out with these same devices. The use of word prediction software was not allowed to limit the bias associated with the type of computer access device on TIS. The speech recognition system was used in a quiet room.

A single evaluation was carried out for each participant. During this evaluation, TIS was evaluated during a copying task involving a 400-word text that the participant was asked to type in $10 \mathrm{~min}$. The 400 -word text was drawn from national newspapers. Average word length was 5.3 characters (s.d. $=0.3$ ). The length of the text was deliberately too long to be copied within $10 \mathrm{~min}$. The evaluation was stopped after $10 \mathrm{~min}$.

\section{Measures}

TIS (words per minute (w.p.m.)) was calculated as follows: number of characters typed in 10 min divided by 10 , including punctuation marks and spaces but not backspace, selection errors or correction times. Results obtained in characters per minute were then divided by 6.3 (the mean number of characters per word in the text+one space) to provide results in w.p.m.

For the speech recognition system, TIS was calculated only on correctly written words (number of characters typed in 10 min divided by 10). Results obtained in characters per minute were divided by 6.3 to provide results in word per minute.

Assessment of participant characteristics. For able-bodied participants, gender, age, level of education, frequency of use of word processing software (regularly: $>$ twice per week; occasionally: $\leqslant$ twice per week) and years of computer use were evaluated using a questionnaire.

For people with cervical SCI, the same characteristics were noted. In addition, injury level, time since lesion, type of computer access device and duration of use of the device were also recorded.

\section{Data analysis}

The data did not follow a normal distribution (Shapiro-Wilk test) $(P=0.04)$. Comparison of able-bodied participants and participants with cervical SCI: descriptive statistics, median and interquartile range (IQR) were used to describe continuous variables, and frequencies were used for categorical variables. A Wilcoxon's test was carried out to analyze differences in age and education level and a $\chi^{2}$ test was used to analyze differences in gender between participants with cervical SCI and able-bodied participants. A Wilcoxon's paired signed-rank test was used to analyze differences in TIS between participants with cervical SCI and able-bodied participants.

Analysis of the characteristics of participants with cervical SCI. The relationship between participant characteristics, computer access device and TIS was analyzed using a Scheirer-Ray-Hare test with the level of lesion as the first factor and, respectively, age, gender, laterality, level of education, time since SCI, duration of use of computer, word processing system used, type of computer access device and the duration of use of access device as the second factors.

Comparison of high and low tetraplegia. Differences in the frequency of use of word processing software between participants with high-level tetraplegia and those with low-level tetraplegia were analyzed using a $\chi^{2}$ test. Differences in TIS between participants with high-level tetraplegia and those with low-level tetraplegia were analyzed using a Wilcoxon's unpaired signed-rank test.

All data were analyzed using the $\mathrm{R}$ Project for Statistical Computing, $\mathrm{R}$ (version 3.0.2) software (The R Foundation, Vienna, Austria). The level of significance was fixed at $P<0.05$.

\section{RESULTS}

\section{Participants}

Table 2 shows the general demographic description of the participants with cervical SCI and the able-bodied participants.

Thirty-five participants with cervical SCI were enrolled in this study. In addition, 21 able-bodied people were recruited. There were no significant differences between groups for age $(P=0.44)$, gender $(P=0.93)$ or years of education $(P=0.08)$. 
Seventeen participants with cervical SCI had a high level of lesion (C4 and C5 Asia A or B) (high tetraplegia group) and 18 participants had lesions between C6 and C8 Asia A or B (low tetraplegia group). In the high tetraplegia group, 10 participants used word processing programs regularly ( $>$ twice per week) and 7 did not $(\leqslant$ twice per

Table 2 Demographic description of participants

\begin{tabular}{lcc}
\hline & Number & Median (IQR) \\
\hline People with cervical SCI & & \\
$\quad$ Gender & 28 & \\
$\quad$ Male & 7 & \\
$\quad$ Female & & $36(32 ; 46)$ \\
Age & & $14(14 ; 17)$ \\
Education years & 17 & \\
Tetraplegia level & 18 & \\
$\quad$ High & & $4(2 ; 11)$ \\
$\quad$ Low & & $16(10 ; 26)$ \\
Time since SCl (years) & $6(1.5 ; 12)$ \\
Years of computer use & & \\
Duration of use of the access device (years) & & \\
Able-bodied people & & \\
Gender & & \\
$\quad$ Male & 17 & \\
$\quad$ Female & 4 & \\
Age & & \\
Education years & & $14(12 ; 15)$ \\
\hline
\end{tabular}

Abbreviations: IQR, interquartile range; $\mathrm{SCl}$, spinal cord injury. week). In the low tetraplegia group, 6 participants used word processing programs regularly and 12 did not (Table 3$)(P=0.002)$.

Table 3 shows the gender and number of years of education for each participant with cervical SCI and the devices they used.

\section{Text input speed}

Median TIS was $9(6 ; 14)$ w.p.m. for participants with cervical SCI and 19 (14; 24) w.p.m. for able-bodied participants $(P=0.001)$.

In the high tetraplegia group, median TIS was $12(4 ; 13)$ w.p.m. In the low tetraplegia group, median TIS was $10(9 ; 18)$ w.p.m. $(P=0.38)$.

\section{Influence of participant characteristics on TIS}

Table 4 shows the TIS of participants with cervical SCI as a function of their characteristics.

For participants with cervical SCI, the Scheirer-Ray-Hare test showed (i) no significant effect on TIS by any characteristic (age $(P=0.76)$, gender $(P=0.47)$, laterality $(P=0.35)$, education $(P=0.06)$, time since SCI $(P=0.92)$, duration of computer use ( $P=0.74)$, word processing use $(P=0.39)$ and duration of use of access device $(P=0.90)$ ); (ii) no significant effect of the level of lesion; and (iii) no significant interaction between any characteristic and the level of lesion.

Influence of computer access device on TIS

Table 5 shows the TIS of participants with cervical SCI as a function of tetraplegia level and computer access device.

For participants with cervical SCI, The Scheirer-Ray-Hare test showed an effect of computer access device $(P<0.001)$, no effect of

Table 3 Tetraplegia level, computer access devices and frequency of use of word processing software by participants with cervical SCI

\begin{tabular}{|c|c|c|c|c|c|}
\hline Number of participants & Gender & Tetraplegia level & Education years & Devices & Frequency of use of word processing \\
\hline 2 & M & High & 17 & Speech recognition system & Regular \\
\hline 2 & M & High & 17 & On-screen keyboard and head pointing & Regular \\
\hline 1 & M & High & 17 & Standard keyboard and mouthstick & Regular \\
\hline 1 & M & High & 12 & Speech recognition system & Regular \\
\hline 1 & M & High & 14 & Speech recognition system & Occasional \\
\hline 2 & M & High & 14 & On-screen keyboard and head pointing & Occasional \\
\hline 1 & M & High & 12 & On-screen keyboard and head pointing & Regular \\
\hline 1 & $\mathrm{~F}$ & High & 17 & On-screen keyboard and head pointing & Regular \\
\hline 1 & M & High & 12 & Standard keyboard & Occasional \\
\hline 1 & M & High & 14 & Standard keyboard & Occasional \\
\hline 1 & M & High & 15 & Standard keyboard & Occasional \\
\hline 1 & M & High & 17 & Standard keyboard & Occasional \\
\hline 1 & $\mathrm{~F}$ & High & 17 & Standard keyboard & Regular \\
\hline 1 & M & High & 12 & Standard keyboard & Regular \\
\hline 1 & $\mathrm{~F}$ & Low & 15 & Speech recognition system & Occasional \\
\hline 4 & M & Low & 14 & Standard keyboard & Occasional \\
\hline 1 & $\mathrm{~F}$ & Low & 17 & Standard keyboard & Occasional \\
\hline 1 & M & Low & 17 & Standard keyboard & Regular \\
\hline 1 & $\mathrm{~F}$ & Low & 14 & Standard keyboard & Regular \\
\hline 1 & M & Low & 14 & Standard keyboard & Regular \\
\hline 2 & M & Low & 15 & Standard keyboard & Regular \\
\hline 2 & $\mathrm{~F}$ & Low & 15 & Standard keyboard & Occasional \\
\hline 1 & M & Low & 19 & Standard keyboard & Occasional \\
\hline 3 & M & Low & 12 & Standard keyboard & Occasional \\
\hline 1 & M & Low & 12 & Standard keyboard & Regular \\
\hline
\end{tabular}


Table 4 TIS with participants with cervical SCI (w.p.m.)

\begin{tabular}{|c|c|}
\hline Participants with cervical SCl & $\begin{array}{l}\text { TIS (w.p.m.), } \\
\text { median (IQR) }\end{array}$ \\
\hline \multicolumn{2}{|l|}{ Gender } \\
\hline M & $9(6 ; 10)$ \\
\hline $\mathrm{F}$ & $15(10 ; 17)$ \\
\hline \multicolumn{2}{|l|}{ Age (years) } \\
\hline $18-40$ & $9(5 ; 16)$ \\
\hline $40-60$ & $9(7 ; 13)$ \\
\hline \multicolumn{2}{|l|}{ Tetraplegia level } \\
\hline High & $12(4 ; 13)$ \\
\hline Low & $10(9 ; 18)$ \\
\hline \multicolumn{2}{|l|}{ Education (years) } \\
\hline$\leqslant 12$ & $8(6 ; 9)$ \\
\hline$\geqslant 13-<15$ & $7(5 ; 7)$ \\
\hline$\geqslant 15$ & $10(10 ; 15)$ \\
\hline \multicolumn{2}{|c|}{ Frequency of word processing program use } \\
\hline Occasionally & $8(6 ; 13)$ \\
\hline Regularly & $10(6 ; 15)$ \\
\hline \multicolumn{2}{|l|}{ Devices } \\
\hline Speech recognition software & $18(17 ; 21)$ \\
\hline On-screen keyboard & $3(3 ; 4)$ \\
\hline Standard keyboard & $8(7 ; 10)$ \\
\hline
\end{tabular}

Abbreviations: IQR, interquartile range; TIS, text input speed; w.p.m., words per minute. Regularly ( $>$ twice per week) and occasionally ( $\leqslant$ twice per week).

Table 5 TIS as a function of tetraplegia level and device used

\begin{tabular}{lclc}
\hline $\begin{array}{l}\text { Tetraplegia level } \\
\text { (AIS A or B) }\end{array}$ & $\begin{array}{c}\text { Number of } \\
\text { persons }\end{array}$ & Computer access device & TIS (w.p.m.), \\
median (IQR)
\end{tabular}

Abbreviations: AIS, ASIA Impairment Scale; IQR, interquartile range; TIS, text input speed; w.p.m., words per minute.

the level of lesion $(P=0.41)$ and no interactions between computer access device and the level of lesion $(P=0.83)$.

\section{DISCUSSION}

The aims of this study were to evaluate TIS in a sample of persons with tetraplegia, and to study the influence of personal characteristics and the type of computer access device on TIS.

This study is the first to provide data regarding TIS in persons with cervical SCI. Moreover, the sample included is the largest in the literature for this type of study. The results showed that TIS was significantly lower for participants with cervical SCI compared with that for able-bodied participants. This is in accordance with results in the literature from studies of persons with a variety of pathologies. ${ }^{9}$

Surprisingly, there was no influence of the characteristics of participants with cervical SCI on TIS. We had hypothesized that characteristics such as education level, duration of computer use and word processing use would influence TIS. In the literature, no studies have evaluated the effect of these characteristics on TIS. The closestrelated study evaluated visuospatial tasks and showed that men performed them better than women. ${ }^{14}$ Another study showed that people with SCI who have a higher level of education use Internet more. ${ }^{16}$ It is possible that a high level of education is related to greater computer use and facilitates the use of Internet. The results of this study, however, suggest that these characteristics do not influence TIS.

The type of computer access device was, however, found to affect TIS, confirming results in the literature. ${ }^{5,7}$ This is likely to be related to the fact that the different types of device are designed for use by persons with different levels of motor ability. For example, the voice recognition system, which is mostly used by persons with high levels of tetraplegia, is actually associated with a higher TIS compared with a virtual keyboard used by persons with lower levels of tetraplegia.

Surprisingly, the results showed that there was no correlation between TIS and lesion level. The most likely explanation is that speech recognition software, used by several participants with high tetraplegia, compensates well for motor difficulties related to the level of injury, leading to a higher TIS. The TIS of participants who used voice recognition software was close to that of able-bodied participants. This would suggest that voice recognition software may be the solution of choice for people with cervical SCI, particularly those with high tetraplegia. However, this result must be interpreted with caution. Participants sat in a quiet room to carry out the evaluation. This situation does not always correspond to real life. For example, in a noisy home environment (with music, television and other people), the use of such software may be compromised. Moreover, voice recognition software requires a high investment in terms of learning time. ${ }^{17}$ After $10 \mathrm{~min}$ of voice recording, it is necessary to learn the most appropriate manner in which to dictate to the software. For example, based on our clinical experience, it is preferable to formulate the sentence mentally before saying it aloud. Similarly, it is necessary to learn command words to correct errors and to navigate within the text. In our experience, this learning period can take from several hours to several days depending on the person. If persons are left to learn alone, they may become discouraged. Indeed, in an unpublished study carried out by our team in 22 participants with cervical SCI, voice recognition software was abandoned by $27 \%$ of users in the first 6 months. In all cases, participants with cervical SCI who abandoned the use of voice recognition software also had another computer access device. Furthermore, we found that the dropout rate for speech recognition software was halved if participants received training. Lack of training and support from health-related professionals is the most cited reason for abandoning this device. Another hypothesis is that people prefer devices that are more adapted for internet use.

Interestingly, the participant with the highest TIS had a C4 A/B SCI. He used a standard keyboard and a mouthstick to input text. This suggests that it may be easier to input text using head movements when the neck muscles are intact, compared with the use of the upper limbs when several upper limb muscles are nonfunctional as in C6, C7 or C8 A/B SCI. The impaired control of the upper limbs may lead to text input errors and a low TIS. However, it is difficult to conclude regarding this point, as the sample only included one participant with high tetraplegia who used this method. 


\section{Limitations}

The results of this study should be interpreted with caution because of the small number of participants in each subgroup with cervical SCI. To avoid even smaller subgroups, we chose not to allow the use of word prediction software during the assessments. Word prediction software can be used with both on-screen and standard keyboards. It is not a computer access device, but it is designed to optimize TIS. However, it may be interesting to study the influence of such software on TIS in future studies. The regular use of word processing by some participants could have increased their text input ability and therefore influenced their TIS. Further studies are necessary to assess the influence of this specific point. However, it must be noted that this study included the largest sample of patients with cervical tetraplegia in the literature.

\section{Supplement material area}

The data did not follow a normal distribution, and we therefore used the Scheirer-Ray-Hare test (nonparametric equivalent of the two-way analysis of variance). However, our data were close to a normal distribution and analysis of variance are known to be 'robust' to violations of the normality assumption. Therefore, sample data might deviate considerably from normality but the test will still yield an appropriate conclusion regarding the null hypothesis ${ }^{18,19}$. Therefore, the results of the present experiment were verified using a two-way analysis of variance with the level of lesion as the independent factor and age, gender, laterality, level of education, time since SCI, duration of computer use, word processing system used, computer access device used and the duration of use of the access device as dependent factors. The results of this complementary analysis yielded the same results as the Scheirer-Ray-Hare test. We are thus confident that our results are robust.

\section{CONCLUSION}

This is the first study to determine TIS in participants with cervical SCI and to evaluate the effect of participant characteristics on TIS. Our results showed that no characteristics influenced TIS, not even the level of lesion. However, the type of computer access device did influence TIS. TIS was highest for participants who used speech recognition systems; however, this device has some drawbacks. We propose that prescription of this device should be more widespread; however, users should be given appropriate training to gain the benefits of the system. It may be useful to advise patients to use several types of access devices depending on their computer needs. It is important to spend time to choose the most appropriate device for each individual to improve TIS, as the results of this study showed that the type of device influenced TIS. Equally, future studies of TIS should take the type of access device into account, rather than the level of the lesion.

The results of this study provide reference data of TIS for patients with cervical SCI, which can be used as a base for further studies on computer use in people with cervical SCI, such as the evaluation of rehabilitation methods.

\section{DATA ARCHIVING}

There were no data to deposit.

\section{CONFLICT OF INTEREST}

The authors declare no conflict of interest.

\section{ACKNOWLEDGEMENTS}

We sincerely thank all those who participated in this study. This research is supported by the Paul Bennetot Fondation (Matmut Group Foundation).

1 Folan A, Barclay L, Cooper C, Robinson M. Exploring the experience of clients with tetraplegia utilizing assistive technology for computer access. Disabil Rehabil Assist Technol 2015; 10: 46-52.

2 Laffont I, Biard N, Bouteille J, Pouplin S, Guillon B, Bernuz B et al. Tétraplégie: solutions technologiques de compensation des incapacités découlant de l'atteinte des membres supérieurs. La Lett Méd Phys Réadapt 2008; 24: 113-121.

3 Guttmann L. Spinal Cord Injuries, Comprehensive Management and Research, 1st ed. Blackwell Science: Oxford, UK, 1973.

4 Betke M, Gips J, Fleming P. The camera mouse: visual tracking of body features to provide computer access for people with severe disabilities. IEEE Trans Neural Syst Rehabil Eng 2002; 10: 1-10.

5 Dalton JR, Peterson CQ. The use of voice recognition as a control interface for word processing. Occup Ther Heal care 1997; 11: 75-81.

6 Koester HH, Levine SP. Effect of a word prediction feature on user performance. Augment Altern Commun 1996; 12: 155-168.

7 Lau C, Leary SO. Comparison of computer interface devices for persons with severe physical disabilities. Am J Occup Ther 1993; 47: 1022-1030.

8 Devries RC, Deitz J, Mailing W. A comparison of two computer access systems for functional text entry. Am J Occup Ther 1998; 52: 656-665.

9 Le Pévédic B. Prédiction Morphosyntaxique Évolutive HandiAS. Thesis, Nantes, France, 1997.

10 Pouplin S, Robertson J, Antoine J, Blanchet A, Kahloun JL, Engineer D et al. Effect of dynamic keyboard and word-prediction systems on text input speed in persons with functional tetraplegia. J Rehabil Res Dev 2014; 51: 467-480.

11 Kim D, Lee B, Lim SE, Kim D, Hwang S, II, Yim Y et al. The selection of the appropriate computer interface device for patients with high cervical cord injury. Ann Rehabil Med 2013; 37: 443-448.

12 Koester HH, Lopresti E, Simpson RC. Toward automatic adjustment of keyboard settings for people with physical impairments. Disabil Rehabil Assist Technol 2007; 2: 261-274.

13 Stronge AJ, Rogers WA, Fisk AD. Web-based information search and retrieval: effects of strategy use and age on search success. Hum Factors 2006; 48: 434-446.

14 Cherney I. Sex differences in effects of testing medium and response format on a visuospatial task. Percept Mot Skills 2010; 110: 809-824.

15 Wobbrock JO, Myers BA, Hall MG. In-Stroke Word Completion. Human-Computer Interact Institute: Montreux, Switzerland, 2006.

16 Matter B, Feinberg M, Schomer K, Harniss M, Brown P, Johnson K. Information needs of people with spinal cord injuries. J Spinal Cord Med 2009; 32: 545-554.

17 Koester $\mathrm{HH}$. Usage, performance, and satisfaction outcomes for experienced users of automatic speech recognition. J Rehabil Res Dev 2004; 41: 739.

18 De Carlo L. On the meaning and use of kurtosis. Psychol Methods 1997; 2: 292-307.

19 West S, Finch JF, Curran PJ. Structural equation models with non-normal variables: problems and remedies. In: Hoyle RH (ed). Structural Equation Modeling: Concepts, Issues and Applications. SAGE Publications Inc: Thousand Oaks, CA, USA, 1995.

20 Mackenzie IS. SAK : scanning ambiguous keyboard for efficient one-key text entry. ACM Trans Comput Interact 2010; 17: 1-39.

21 Mankowski R, Simpson RC, Koester HH. Validating a model of row-column scanning. Disabil Rehabil Assist Technol 2013; 8: 321-329.

22 Garrett JT. Using speech recognition software to increase writing fluency for individuals with physical disabilities. J Special Educ Technol 2011; 26: 25. 